\title{
Erratum: Global Behavior of 1d-Viscous Compressible Barotropic Fluid with a Free Boundary and Large Data
}

Ivan Straškraba and Alexander Zlotnik

After publishing our paper [1], two defects have been found in the proof of Theorem 3.2. We shall show that in fact this theorem along with the following Theorem 3.3 and Corollary 3.1 are valid under additional condition

$$
f_{\infty}(x) \leq 0 \quad \text { in } \quad B\left(\ell_{\infty}\right):=\left(\ell_{\infty}-\delta_{c}, \ell_{\infty}+\delta_{c}\right)
$$

for sufficiently small $\delta_{c}>0$. First, since in fact

$$
\frac{d}{d t} \int_{\Omega_{\infty}}\left(p_{\Gamma, \infty}-p\left(\rho_{\infty}\right)\right) d x=0
$$

(namely with $\Omega_{\infty}$ instead of $\Omega_{t}$ as on p. 136), the summand $\frac{d}{d t} \mathcal{A}$ with

$$
\mathcal{A}(t):=\int_{\ell_{\infty}}^{\ell(t)}\left(p_{\Gamma, \infty}-p\left(\rho_{\infty}\right)\right) d x
$$

was lost in the left-hand sides of equalities (3.21) and (3.24). Consequently the summand $\mathcal{A}$ should be included into the Lyapunov functional $\mathcal{V}_{\varepsilon}$. Under condition (1), $p\left(\rho_{\infty}\right)$ is nonincreasing in $B\left(\ell_{\infty}\right)$ and since also $p_{\Gamma, \infty}=p\left(\rho_{\infty}\left(\ell_{\infty}\right)\right)$, we have

$$
0 \leq \mathcal{A} \leq\left[p_{\Gamma, \infty}-p\left(\rho_{\infty}(\ell)\right)\right]\left(\ell-\ell_{\infty}\right) \leq K\left(\ell-\ell_{\infty}\right)^{2}
$$

for $t \geq t_{0}$

Next, clearly

$$
\left(\pi(\rho)-\pi\left(\rho_{\infty}\right)\right)_{\ell}=\pi\left(\rho_{\ell}\right)-\pi\left(\rho_{\infty}\left(\ell_{\infty}\right)\right)+\pi\left(\rho_{\infty}\left(\ell_{\infty}\right)\right)-\pi\left(\rho_{\infty}(\ell)\right) .
$$

Since $\pi\left(\rho_{\infty}\right)$ is also nonincreasing in $B\left(\ell_{\infty}\right)$ and Remark 3.3 holds, we get

$$
\begin{aligned}
& -\left[\pi\left(\rho_{\infty}\left(\ell_{\infty}\right)\right)-\pi\left(\rho_{\infty}(\ell)\right)\right]\left(I\left(\rho-\rho_{\infty}\right)\right)_{\ell} \\
& =\left[\pi\left(\rho_{\infty}\left(\ell_{\infty}\right)\right)-\pi\left(\rho_{\infty}(\ell)\right)\right] \int_{\ell_{\infty}}^{\ell} \rho_{\infty} d x \geq 0
\end{aligned}
$$

for $t \geq t_{0}$. The estimate for the boundary term in the functional $\mathcal{W}_{\varepsilon}$ given on p. 137 is valid in fact with $\pi\left(\rho_{\infty}\left(\ell_{\infty}\right)\right)$ instead of $\pi\left(\rho_{\infty}\right)$. Therefore finally taking 
into account (2) and (3), we can assert that relations (3.25)-(3.27) are valid under condition (1) and so Theorem 3.2 does.

Notice also that in the formula for $\mathcal{E}^{\prime}$ on p. 125 the term $p(\rho)$ should be replaced by $p(\rho)-p_{\Gamma, \infty}$ and in estimate $(3.32) \rho_{\infty}\left(\ell_{\infty}\right)$ should be replaced by $\rho_{\infty}(\ell)$ which do not change the results (concerning (3.32), this follows from the equality $\rho_{\ell}-$ $\rho_{\infty}(\ell)=\rho_{\ell}-\rho_{\infty}\left(\ell_{\infty}\right)+\rho_{\infty}\left(\ell_{\infty}\right)-\rho_{\infty}(\ell)$ along with Remark 3.3).

In a forthcoming paper, it will be shown that condition (1) can be essentially enlarged.

\section{References}

[1] I. Straškraba And A. Zlotnik, Global behavior of 1d-viscous compressible barotropic fluid with a free boundary and large data, J. Math. Fluid. Mech. 5 (2003), 119-143.

I. Straškraba

Mathematical Institute

Academy of Sciences

Žitná 25

11567 Praha 1

Czech Republic

e-mail: strask@math.cas.cz
A. Zlotnik

Department of Mathematical Modelling

Moscow Power Engineering Institute

Krasnokazarmennaja 14

111250 Moscow

Russia

e-mail: zlotnik@apmsun.mpei.ac.ru

(received by the Editors: February 10, 2004)

(17) To access this journal online:

(40) http://www.birkhauser.ch 